\title{
Insights into the miRNA regulations in human disease genes
}

\author{
Jyotirmoy Das, Soumita Podder ${ }^{*}$ and Tapash Chandra Ghosh ${ }^{*}$
}

\begin{abstract}
Background: MicroRNAs are a class of short non-coding RNAs derived from either cellular or viral transcripts that act post-transcriptionally to regulate mRNA stability and translation. In recent days, increasing numbers of miRNAs have been shown to be involved in the development and progression of a variety of diseases. We, therefore, intend to enumerate miRNA targets in several known disease classes to explore the degree of miRNA regulations on them which is unexplored till date.

Results: Here, we noticed that miRNA hits in cancer genes are remarkably higher than other diseases in human. Our observation suggests that UTRs and the transcript length of cancer related genes have a significant contribution in higher susceptibility to miRNA regulation. Moreover, gene duplication, mRNA stability, AREScores and evolutionary rate were likely to have implications for more miRNA targeting on cancer genes. Consequently, the regression analysis have confirmed that the AREScores plays most important role in detecting miRNA targets on disease genes. Interestingly, we observed that epigenetic modifications like CpG methylation and histone modification are less effective than miRNA regulations in controlling the gene expression of cancer genes.
\end{abstract}

Conclusions: The intrinsic properties of cancer genes studied here, for higher miRNA targeting will enhance the knowledge on cancer gene regulation.

Keywords: MicroRNAs, Cancer, mRNA decay rates, AREScores, Epigenetic modifications

\section{Background}

MicroRNAs (miRNAs) are abundant classes of endogenous small non-coding RNAs approximately 2123 nucleotides (nts) long transcripts generated from 70-100 nts hairpin precursors, which regulate gene expression post-transcriptionally by affecting the translation of target messenger RNAs (mRNAs) [1]. These small miRNA molecules play important roles in cell growth, differentiation, proliferation, apoptosis, and polarization of neurons [2]. With the recent advancement of experimental studies, many biological factors have been revealed to contribute in the recognition of miRNA targeting [3]. Indepth characterization of miRNA targets enables better understanding of the role of miRNAs in various biological processes.

There is a growing body of evidence regarding the importance of miRNAs in human diseases [4]. Studies have established that mutations, dysregulations or dysfunction

\footnotetext{
*Correspondence: soumita@jcbose.ac.in; tapash@jcbose.ac.in Bioinformatics Centre, Bose Institute, P 1/12, C.I.T. Scheme VII M, Kolkata 700 054, India
}

of miRNA biogenesis and their targets led to the blockage of physiological and biochemical pathways that influenced various diseases in human [5]. Computational prediction did not only reveal a number of miRNAdisease associations but also showed that the mechanistic associations in miRNAs and human diseases are very complicated. Previously, it has also been demonstrated that genetic defects in miRNAs, their processing machinery and epigenetic regulations are common hallmarks of human diseases [3].

Although a number of studies have dealt with the miRNA-disease association, but no study has yet been conducted in concerning the type of disease class which is more susceptible to miRNA target. In the present study, we aimed to identify the disease class which is more prone to miRNA target. Our studies indicated that the cancer disease genes are more prevalent in miRNAtargeted human disease genes compared to the other disease gene classes. These findings raise the obvious question, why cancer genes are more targeted by miRNAs. To explain this, we have analyzed the role of various gene 
subcomponents, mRNA decay rates, mRNA stability, AREScores. Finally, we revealed a remarkable insight that the miRNAs have more influence than epigenetic modifications in controlling the expression of cancer related genes.

\section{Results}

\section{miRNA targets of disease genes}

We have separated human disease genes depending on the miRNA target to the disease genes in two groups: miRNA-targeted disease genes and miRNA nontargeted disease genes. We aimed to find out the disease class which is mostly targeted by miRNA among all disease classes. Thus, we have broadly categorized a total of 301 miRNA targeted disease genes into eight different disease classes according to the Human Gene Mutation Database (HGMD) [6], such as cancer disease genes, neurological disease genes, developmental disease genes, metabolic disease genes, respiratory tract disease genes, immunological disease genes, cardiovascular disease genes and muscle/bone disease genes. The miRNA targeted disease genes category and their corresponding miRNA targets are listed in the online supplement (Additional file 1: Table S1). Measuring the miRNA target profile across the disease gene classes, we have noticed significant differences in their distributions (Figure 1). Among all the disease classes, cancer genes $(29.80 \%)$ were observed to be mostly targeted by miRNA (Figure 1a). Moreover, we have noticed the average number of miRNA target sites were also higher for cancer genes compared to the other classes of genes (Figure 1b). To exemplify the novel characteristics of cancer genes required for mRNA::miRNA base-pairing, we have merged the other seven disease classes into non-cancer disease group and performed the rest of the study by comparing cancer $(\mathrm{N}=90)$ and non-cancer disease genes $(\mathrm{N}=211)$ (Additional file 1: Table S1).

\section{Gene subcomponents in cancer genes}

It was evident that miRNAs perform their regulatory roles mainly by base-pairing with 3'-UTRs of target genes. Therefore, genes targeted by miRNAs should have longer 3 '-UTR sequences [7]. Hence, we have calculated lengths of 3 '-UTRs and observed that the average 3 'UTRs length are longer for cancer genes than non-cancer disease genes (Table 1). To determine the effect of other gene subcomponents, we have calculated the length of $5^{\prime}$ UTRs and transcript of these two disease groups since it was ascertained that miRNA also targets at these regions [8]. We have found that both $5^{\prime}$-UTRs lengths and transcript lengths are significantly higher on average for cancer disease genes compared to non-cancer disease genes (Table 1). To understand the relationship between gene subcomponents and number of miRNA hits, we performed correlation analysis [7,9]. Considering all the disease genes together, we have noticed that both the $3{ }^{\prime}$ - and $5^{\prime}$-UTRs as well as transcript length hold significant positive correlations with the number of miRNA hits $\left(\rho_{3^{\prime} \text {-UTR }}\right.$ vs. miRNA hits $=0.532 ; P=2.2 \times 10^{-19}, N=243 ; \rho_{5^{\prime} \text {-UTR vs. }}$ miRNA hits $=0.153 ; P=4.9 \times 10^{-2}, N=212 ; \rho_{\text {transcript length }}$ vs. miRNA hits $=0.166 ; P=4.2 \times 10^{-2}, N=150$ ).

Therefore, it could be expected that longer structure of genes is most likely to have more complex regulation. Indeed, we have found that longer gene structures (Table 1) is significantly higher in cancer genes than non-cancer disease genes, indicating that gene lengths are more crucial for miRNA regulation of cancer genes (see Additional file 2: Table S2).

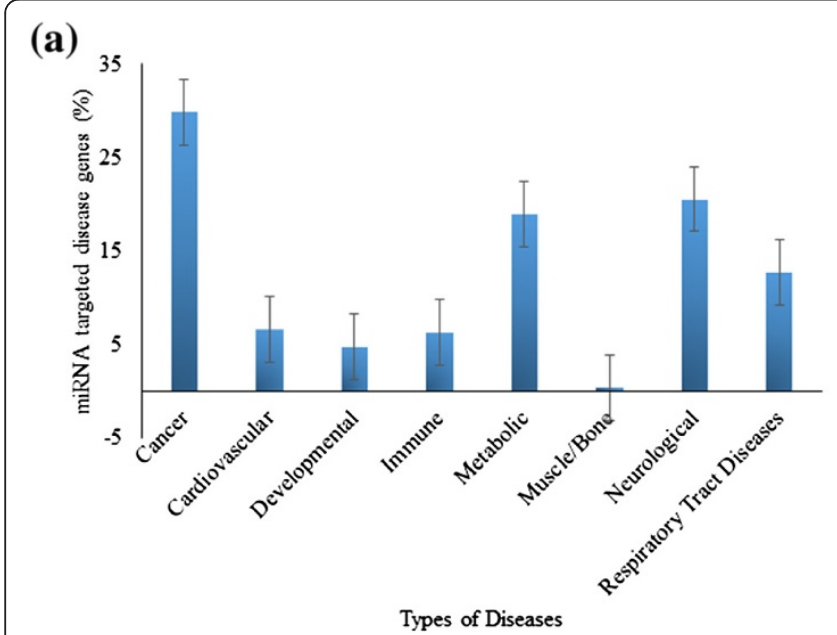

(b)

Figure 1 (a) Distribution of miRNA targeted disease genes (in percentage) (Error bar indicates 5\% standard error). (b) Average number of miRNA hits among cancer and non-cancer disease genes (Error bar indicates 5\% standard error). 


\begin{tabular}{|c|c|c|c|}
\hline $\begin{array}{l}\text { Structural } \\
\text { parameters }\end{array}$ & $\begin{array}{c}\text { miRNA-targeted } \\
\text { cancer disease } \\
\text { genes (bp) }\end{array}$ & $\begin{array}{c}\text { miRNA-targeted } \\
\text { non-cancer disease } \\
\text { genes (bp) }\end{array}$ & $\begin{array}{c}\text { Level of } \\
\text { significance } \\
(P) \\
\end{array}$ \\
\hline $\begin{array}{c}\text { Average } \\
\text { 3'-UTR lengths }\end{array}$ & 1143 & 908 & $4.4 \times 10^{-2}$ \\
\hline $\begin{array}{c}\text { Average } \\
\text { 5'-UTR lengths }\end{array}$ & 349 & 254 & $4.2 \times 10^{-2}$ \\
\hline $\begin{array}{c}\text { Average } \\
\text { transcript lengths }\end{array}$ & 4196 & 3418 & $2.8 \times 10^{-2}$ \\
\hline
\end{tabular}

\section{Intrinsic genomic properties of cancer genes}

To reveal the pattern of gene expression level of the miRNA-targeted cancer genes, we computed the correlation between miRNA hits and gene expression levels of cancer and non-cancer disease genes separately. We obtained a significant negative correlation between miRNA hits and the expression levels of cancer disease genes $\rho_{\text {expression levels vs. miRNA hits }}=-0.266 ; P=3.8 \times 10^{-2}$; $N=51)$. However, no significant correlation exists in the case of non-cancer disease genes $\left(\rho_{\text {expression levels vs. miRNA }}\right.$ hits $=0.004 ; P=9.9 \times 10^{-1} ; N=122$ ). Moreover, we have noticed that the percentage of lowly expressed genes are much higher in miRNA-targeted cancer disease genes than non-cancer disease genes (in cancer: $49.18 \%$; in noncancer: $31.97 \%$; at 95\% significance level) which suggests that miRNAs prefer the lowly expressed genes for their target. This observation was also echoed in an earlier report that proposed highly expressed genes could not be targeted by miRNA due to their shorter genomic structure [7].

Next, to examine whether the higher expression of onco-miRNAs repressed the expression of onco-mRNAs, we did correlation analysis between miRNA expression and mRNA expression of cancer genes. We have obtained a negative correlation between them $\left(\rho_{\text {mRNA expression vs. }}\right.$ miRNA expression $=-0.273 ; \quad P=3.9 \times 10^{-2} ; \quad N=51$ ) (Additional file 3: Figure S1). This result clearly indicates that miRNA plays a significant role in lowering the expression of cancer genes.

Conrad et al. [10] showed that duplicate genes play important roles in human diseases and it has also been reported that miRNA is prerequisite for dosage compensation of duplicated genes [11]. Thus, we have tested the prevalence of gene duplications in miRNA-targeted cancer and non-cancer genes and observed that gene duplication are more frequent in cancer genes than noncancer genes (duplicate frequency in cancer: $58.24 \%$; in non-cancer: $45.02 \%$; at 95\% significance level, $N=301$ ) (Additional file 3: Figure S2).

Gene duplication is the key regulatory mechanism of genome and organism evolution. It was already reported that genes having more distinct miRNA binding sites at the 3'UTR regions tend to have slower evolutionary rates [9]. Measuring evolutionary rates of the cancer and non-cancer genes, we have noticed that evolutionary rates are slower for miRNA-targeted cancer genes than non-cancer genes (in cancer: 0.1786; in noncancer: $0.3129 ; P=3.0 \times 10^{-6}, N=281$ ) (Additional file 3: Figure S3).

Our observation is in accordance with the report of Thomas et al. (2003) [12]. Thus, higher purifying selection on cancer genes could be treated as a trademark of higher miRNA targets.

\section{Mode of cancer genes regulation by miRNA}

Generally, in a cellular environment, miRNAs mediate gene regulation by reducing the stability of their target mRNAs through mRNA degradation methods or using translational repression processes [7]. Thus, we hypothesized that the cancer genes should have more mRNA decay rates than others. Therefore, to test mRNA stability differences between miRNA-targeted cancer disease genes and miRNA-targeted non-cancer genes on the genomic scale, we have compared mRNA decay rates which were taken from Pai et al. [13]. We have noticed a significant positive correlation between miRNA targets and mRNA decay rates $\left(\rho_{\text {miRNA }}\right.$ hits vs. mRNA decay rates $=0.119 ; P=5.0 \times 10^{-2} ; N=264$ ) (Additional file 3 : Figure S4). This result suggests that the number of miRNA targets may enhance mRNA decay rates which is in agreement with the previous findings that miRNA targeted genes have higher decay rates [7].

Jing et al. [14] showed that the ARE-(AU-rich elements) containing mRNA degradation required to be targeted by miRNAs. Therefore, we have calculated the correlation between miRNA targets and AREScore and found a positive correlation between them $\left(\rho_{\text {miRNA hits vs. }}\right.$ AREScore $=0.124 ; P=4.0 \times 10^{-2} ; N=273$ ) (Additional file 3: Figure S5). Higher miRNA targets of cancer genes may be the artifact of higher AU-rich element in cancer genes. Thus, we proposed that cancer genes regulation mediated through miRNA is achieved via lowering the stability of corresponding mRNA.

\section{Relative contribution of the factors in determining miRNA} targets on disease genes

We have studied several factors that might influence the miRNA targets on disease genes. In this section we summarize the relative contribution of each of these factors that contribute significantly for determining miRNA targets on genes. Since, gene duplication data is a binary variable, we have converted rest of the factors into the binary variables and performed logistic regression analysis by taking number of miRNA hits as a dependent variable and gene subcomponents, gene duplication, evolutionary rate, mRNA decay rate, AU-rich 
elements (AREScores) as the independent variables. We did not consider epigenetic modifications since they did not show any significant effects on miRNA hits in correlation analysis. The result delineated in Table 2 strongly advocates that AREScores is the most important predictor of miRNA hits. The relative contribution of the factors is in the order of AREScores > 3'-UTR lengths $>$ transcript lengths $>5^{\prime}$ - UTR lengths.

\section{miRNA regulations vs. epigenetic modifications of cancer genes}

Proper orchestration of gene expression primarily depends on the epigenetic modifications and miRNA regulation [15]. A close association between epigenetic modifications and miRNAs is just beginning to be understood and has a great importance in the field of molecular biology. Recent studies suggested that miRNAs are considered as the important players in the regulation of epigenetic modifications, such as DNA methylations and histone modifications [16]. We, therefore, intended to investigate whether epigenetic modifications or miRNA plays the most important role in regulating cancer genes expression. For this purpose, we have measured two important epigenetic modifications like CpG methylation and histone modifications in cancer and non-cancer disease genes and observed that the cancer genes have relatively lower level of methylation $(68.13 \%)$ as compared to non-cancer disease genes (82.94\%) (Significant at 99\% level, McCullum Proportion test). We did not find any significant correlation ( miRnA hits vs. $\mathrm{CpG}$ methylation $=0.115, P=$ $7.7 \times 10^{-1}, N=237$ ) between miRNA hits and CpG methylation which suggests that gene regulation by miRNA and CpG methylation is not interconnected. Histone modifications are another part of epigenetic silencing mechanism in mammals. It was previously reported that non-coding RNAs can direct the cytosine methylation and histone modifications that are related

Table 2 The relative contributions of different parameters on number of miRNA targets

\begin{tabular}{ccc}
\hline Variables & $\boldsymbol{\beta}$ value & Level of significance \\
\hline ARE Scores & 2.712 & $2.5 \times 10^{-4}$ \\
3'-UTR lengths & 1.914 & $3.4 \times 10^{-3}$ \\
Transcript lengths & 1.695 & $1.0 \times 10^{-2}$ \\
5'-UTR lengths & 1.562 & $3.4 \times 10^{-2}$ \\
Intron lengths & 0.335 & $7.1 \times 10^{-1}$ \\
CDS lengths & -0.090 & $9.0 \times 10^{-1}$ \\
Gene lengths & -0.151 & $8.3 \times 10^{-1}$ \\
Paralogs & -0.157 & $8.4 \times 10^{-1}$ \\
mRNA decay rates & -0.287 & $6.4 \times 10^{-1}$ \\
Evolutionary Rates & -0.939 & $1.6 \times 10^{-1}$ \\
\hline
\end{tabular}

to gene expression regulation in complex organisms among several other unrelated functions [17]. Thereafter, we have computed the histone modification to determine its effect on cancer and non-cancer genes. However, we did not obtain any significant difference in the rate of histone modification between cancer and non-cancer disease genes (in cancer: 677.8118; in non-cancer: 693.5784; $P=9.8 \times 10^{-1}, N=289$ ). Here also we did not get any significant relation between miRNA hits and histone modification $\left(\rho_{\text {miRNA }}\right.$ hits vs. histone modification $=0.088, P=1.4 \times 10^{-1}$, $N=289)$.

To analyze the effective roles of miRNA hits and epigenetic modifications on disease gene expression, we performed a partial correlation analysis by considering these factors (i.e., gene expression, miRNA hits, histone modifications, and DNA methylation) and found that disease gene expression is negatively correlated with miRNA hits when both epigenetic modifiers i.e. CpG methylation and histone modifications are controlled (Table 3), but the correlation between gene expression and CpG methylation disappeared when we controlled the miRNA hits and histone modifications. Moreover, we also did not find any significant correlation between gene expression and histone modifications after controlling CpG methylation and miRNA hits (Table 3).

These results suggest that miRNAs play more significant role in controlling the disease gene expression than epigenetic modifications such as $\mathrm{CpG}$ methylations or histone modifications.

\section{Discussion}

In this manuscript, we have sought to address three questions: First, which class of disease genes is most targeted by miRNAs? Second, why is this particular class of disease genes frequently targeted by miRNAs? Third, whether miRNAs or epigenetic modifications is the major guiding factor in controlling the expression of disease genes in human? We have detected that miRNAs commonly targets the cancer disease genes. To explore the underlying aims of this observation, we have analyzed several structural and functional parameters. We have traced the important role of 3 '-UTRs among other gene subcomponents in determining the miRNA target site in cancer disease genes. miRNAs usually target genes which have long 3'-UTRs, and cancer genes are observed to possess longer UTRs than non-cancer disease genes. Therefore, it is quite reasonable that miRNA readily target the cancer disease genes. We also tested gene expression levels and noticed that lowly expressed genes are abundant in miRNA targeted cancer genes. Consequently, we also observed that miRNA targeted cancer genes are evolutionary slower than miRNA targeted non-cancer disease genes. Since miRNA targeted genes are proposed to be evolutionary slower [7], the 
Table 3 Partial correlation analysis for miRNA-targeted cancer gene expression with miRNA hits, CpG methylation and histone modifications

\begin{tabular}{ccc}
\hline Factors & Partial correlation for disease gene expression & Level of Significance \\
\hline Number of miRNA hits & $-\mathbf{0 . 3 4 5}$ (controlling CpG methylation ratio and histone modification rate) & $1.6 \times 10^{-2}$ \\
CpG methylation ratio & $\mathbf{0 . 1 6 9}$ (controlling histone modification and miRNA hits) & $2.5 \times 10^{-1}$ \\
Histone modification rate & $\mathbf{0 . 1 4 3}$ (controlling miRNA hits and CpG methylation ratio) & $3.3 \times 10^{-1}$ \\
\hline
\end{tabular}

slower evolutionary rate of cancer genes could be treated as a good substrate for miRNA target compared to noncancer disease genes. However, the relation between expression and evolutionary rate pursued in our study may arise a controversy since evolutionarily conserved genes are evident to have higher expression levels [18]. However, it is also reported that highly expressed genes are generally linked with lower mRNA decay rates, and genes undergoing rapid mRNA decay, are enriched with putative binding sites for miRNA and RNA binding proteins [13]. In our case, we observed that the cancer genes have higher mRNA decay rates due to higher AUrich elements and miRNA target sites. So, higher mRNA decay rates may bring down the expression of miRNA targeted cancer genes. Moreover, the reduction of mRNA levels by miRNAs in cancer genes also suggested the reasonable hypothesis that miRNAs in some cases could stimulate mRNA decay through increasing decapping rates [19]. The longer gene structure of poorly expressed miRNA targeted cancer genes also supports the 'selection for economy' theory that explains the highly and broadly expressed genes have to be shorter to reduce the high energy cost for transcription [20]. This observation also implies that the expression of miRNA targeted cancer genes cannot be attributed to their slower evolutionary rate. This could be treated as an exceptional case where expression could not explain the reason for slower evolutionary rates. It may be the selective stringency of miRNA targeted genes since they possess longer 3 '-UTR structure which is reported to hold strong negative correlation with evolutionary rates [9], or it may be the integral property of cancer disease genes as they are over-represented in the collection of essential genes [12] which are known to be evolutionarily conserved [21]. In addition, intense purifying selection may help to prevent multigene interactions concerned in certain cancers [12]. Moreover, higher duplicability of miRNA targeted cancer genes observed in our study was also relevant to their slower evolutionary rates.

Now the question remains, miRNA targets or epigenetic modifications which one controls the gene expression in miRNA-targeted cancer disease genes. In our study, we noticed that the epigenetic regulation is less likely to control the expression of cancer genes compared to miRNA regulations. Occasionally, miRNA also gets deregulated by several mechanisms like inefficient processing of miRNAs through drosha or dicer etc. This epigenetic silencing of miRNAs in cancer cells modulates the activity of oncogenes as well as the tumor suppressor genes [22]. So, it is quite natural if miRNA gets deregulated, it will induce disease phenotypes. In our result, we found that cancer disease is more prevalent than non-cancer disease genes in miRNA deregulated disease gene set (average number of miRNA targets: in cancer = 4.0222; in non-cancer $=1.7488, P=1.0 \times 10^{-14}, N=301$ ). Persistence of human miRNA genes in the genomic regions involved in the loss of heterozygosity, amplification or breakpoints in cancers [18], also suggesting a link between miRNA and the development of cancer.

\section{Conclusions}

In summary, we have reported ample evidences to support the link between longer gene structure, higher enrichment of AREs, higher duplicability, slower evolutionary rates, and lower mRNA decay rates of cancer genes that make them good substrate for miRNA targeting. Regression analysis has established AU-rich elements as the most influential genomic property that determines miRNA hits on disease genes. The cause and fate of miRNA targets on cancer genes analyzed in our study will help in enhancing the knowledge and medicinal improvement of cancer genes.

\section{Methods}

\section{Human disease genes and microRNA data}

Human (Homo sapiens) disease genes were collected from The International Cancer Gene Consortium (ICGC, ftp:// data.dcc.icgc.org/), Human Gene Mutation Database (HGMD, http://www.hgmd.cf.ac.uk/ac/index.php) [6], and Genetic Association Database (GAD, http://geneticassociationdb.nih.gov/) [23]. Disease genes in our study have been classified in eight major disease classes, like cancer, cardiovascular, developmental, immunological, metabolic, muscle/bone disorder, neurological and respiratory tract diseases according to HGMD.

After removing the redundancy in disease gene datasets, we considered only those genes for which miRNA targets information are available in TargetScan release 6.2 database (http://www.targetscan.org/) [24] and DIANA TarBase v6.0 (http://diana.imis.athena-innovation.gr/ DianaTools/index.php) [25]. It was reported that TargetScan have high fidelity for target prediction from 
biological and informatics validation. TargetScan is used for its reported accuracy and advantages of seedpairing mechanisms in miRNA (which is required for mRNA-miRNA bindings) over other miRNA databases. Furthermore, to increase the reliability of our results, we only considered the miRNAs whose target sites are conserved across most mammals (as defined by TargetScan) [24]. We used context+score as defined by Garcia et al. [26]. DIANA TarBase provides experimentally verified data and it is the largest available manually curated target database. In order to achieve more consistent results, we have considered only those miRNA targets those are present in both the prediction database as well as experimental database. Finally, we have collected only a total of 301 miRNA-targets for our analysis (Additional file 1: Table S1).

\section{Gene subcomponent data}

Gene subcomponents, such as 3'-UTR lengths, 5 '-UTR lengths, transcript lengths data were collected from UCSC genome browser (hg19) [27,28] and Ensembl Biomart database v69 [29] (Additional file 2: Table S2).

\section{Gene expression data}

The mRNA expression data collected from GNF Gene Atlas (http://biogps.org/). The expression values were averaged over tissues and sorted into ascending order. Then the values are equally divided into three groups. The first group containing low expressed genes, the second group is of medium expressed genes and the third one contains highly expressed genes.

Next, we have retrieved miRNA expression data from CancerMiner database [30] which contains microarray expression data for ten tissues of cancer patients. We have averaged the expression values over tissues and then mapped the miRNA expression levels with our dataset (Additional file 2: Table S2).

\section{Identification of paralogs}

Human paralogs were downloaded from ENSEMBL database (v69) [29]. We have collected 148 duplicated genes data after removing the redundant entries and taking the paralogous similarity cut-off value $40 \%$ for our data [11] (Additional file 2: Table S2).

\section{Calculation of evolutionary rate}

Protein evolutionary rates $[d \mathrm{~N} / d \mathrm{~S}]$ data for human using 1:1 orthology relationship to Chimpanzee (Pan troglodytes) were downloaded from ENSEMBL database (v69). Next, we mapped the evolutionary rate data to miRNA targeted human disease genes for further analysis (Additional file 2: Table S2).

\section{mRNA decay rate and ARE score data}

A total of 16,823 mRNA decay rate data was obtained from Pai et al. [13] dataset. We have mapped the data with our dataset and finally collected 267 data for our analysis. AREScore data was collected from AREScore database (http://arescore.dkfz.de/arescore.pl) [31] by assigning all default parameter setup (Additional file 2: Table S2).

\section{Collection of epigenetic data}

Histone modification data were retrieved from human histone modification database (HHMD, http://202.97.205.78/ hhmd/index.jsp) [32] which contains useful histone modifications information from experimental data that is essential for understanding the modifications at a systematic level (Additional file 2: Table S2). We have collected DNA methylation data from NGSmethDB (http://bioinfo2.ugr. es/NGSmethDB/index.php) [33] database which is based on next-generation sequencing DNA methylation data from different human tissues (Additional file 2: Table S2).

\section{Statistical analysis}

All statistical analysis except partial correlations was performed using SPSS v20 and R v3.0.2. TANAGRA (v1.4) [34] was used to determine the partial correlation. For correlation analysis, we have used Spearman's rank correlation test since all of our data shows non-parametric distribution. To test the non-parametric distributions of our dataset we have performed Shapiro-Wilk test (used for the dataset smaller than 2000) (Table 4). To find the difference between two datasets, we have performed Mann-Whitney U test (Two-tailed test). The results are considered to be significant if the P-value is less than 0.05 . We used McCullum proportion test to verify the confidence level of the proportion data used in the study.

Table 4 Shapiro-wilk test results to show the non-parametric dataset used in our study

\begin{tabular}{ccc}
\hline Parameters & W score & $\boldsymbol{P}$ value \\
\hline ARE Scores & 0.786 & $1.3 \times 10^{-16}$ \\
3'-UTR lengths & 0.951 & $4.4 \times 10^{-7}$ \\
Transcript lengths & 0.931 & $2.0 \times 10^{-6}$ \\
5'-UTR lengths & 0.785 & $2.6 \times 10^{-15}$ \\
Paralogs & 0.824 & $4.5 \times 10^{-12}$ \\
mRNA decay rates & 0.735 & $2.2 \times 10^{-22}$ \\
Evolutionary Rates & 0.787 & $7.4 \times 10^{-19}$ \\
CpG methylation & 0.891 & $4.5 \times 10^{-12}$ \\
Histone modification rate & 0.699 & $2.0 \times 10^{-22}$ \\
miRNA hits & 0.929 & $7.8 \times 10^{-11}$ \\
mRNA expression levels & 0.193 & $8.3 \times 10^{-27}$ \\
\hline
\end{tabular}




\section{Additional files}

Additional file 1: Table S1. miRNA targeted disease genes category and their corresponding number and name of miRNA hits.

Additional file 2: Table S2. miRNA targeted disease genes and their different characteristics (gene subcomponents, miRNA expression, gene duplication, evolutionary rates, mRNA decay rate, AU rich element, CPG methylation, Histone modifications) analyzed in our study.

Additional file 3: Figures S1. Correlation between microRNA expression levels and microRNA targeted repressed mRNA expression levels in Cancer disease genes. S2. Percentage of duplicated genes in miRNA-targeted cancer and non-cancer disease genes. S3. Mean difference of evolutionary rates of cancer and non-cancer disease genes in human. S4. Correlation between mRNA decay rates and number of miRNA targets. S5. Correlation between $\mathrm{AU}$-rich element scores and number of miRNA targets.

\section{Competing interests}

The authors declare that they have no competing interests.

\section{Authors' contributions}

JD conceived, designed and performed the work. JD wrote the manuscript, SP helped in planning the work and drafting the manuscript and TCG helped in planning and sketching the manuscript. All authors reviewed the manuscript. All authors read and approved the final manuscript.

\section{Acknowledgments}

Grateful thanks are due to the Department of Science and Technology, Government of India for providing DST-INSPIRE (IF-110696) to JD for providing financial support. We are grateful to Professor Giorgio Bernardi for critical reading and valuable suggestions in improving the manuscript. We also like to thank Ms. Tina Begum for her help in statistical analysis and our anonymous reviewers for their suggestions.

Received: 11 July 2014 Accepted: 11 November 2014 Published: 21 November 2014

\section{References}

1. Bartel D: MicroRNAs: genomics, biogenesis, mechanism, and function. Cell 2004, 116(2):281-297.

2. Wienholds $E$, Plasterk R: MicroRNA function in animal development. FEBS Lett 2005, 579(26):5911-5922.

3. Croce C: Causes and consequences of microRNA dysregulation in cancer. Nat Rev Genet 2009, 10(10):704-714.

4. Calin G, Croce C: MicroRNA signatures in human cancers. Nat Rev Cancer 2006, 6(11):857-866.

5. Lu M, Zhang Q, Deng M, Miao J, Guo Y, Gao W, Cui Q: An analysis of human microRNA and disease associations. PLoS One 2008, 3(10):e3420.

6. Stenson PD, Ball EV, Mort M, Phillips AD, Shaw K, Cooper DN: The human gene mutation database (HGMD) and its exploitation in the fields of personalized genomics and molecular evolution. Curr Protoc Bioinformatics 2012, Chapter 1:Unit1.13.

7. Mok Y, Park S, Choi S: Comparative analysis of the structural and expressional parameters of microRNA target genes. Gene 2012, 497(1):103-109.

8. Zhou X, Duan X, Qian J, Li F: Abundant conserved microRNA target sites in the $5^{\prime}$-untranslated region and coding sequence. Genetica 2009, 137(2):159-164.

9. Cheng C, Bhardwaj N, Gerstein M: The relationship between the evolution of microRNA targets and the length of their UTRs. BMC Genomics 2009, 10:

10. Conrad B, Antonarakis S: Gene duplication: a drive for phenotypic diversity and cause of human disease. Annu Rev Genomics Hum Genet 2007, 8:17-35.

11. Das J, Chakraborty S, Podder S, Ghosh T: Complex-forming proteins escape the robust regulations of miRNA in human. FEBS Lett 2013 587(14):2284-2287.

12. Thomas M, Weston B, Joseph M, Wu W, Nekrutenko A, Tonellato P: Evolutionary dynamics of oncogenes and tumor suppressor genes: Higher intensities of purifying selection than other genes. Mol Biol Evol 2003, 20(6):964-968.
13. Pai A, Cain C, Mizrahi-Man O, De Leon S, Lewellen N, Veyrieras J, Degner J, Gaffney D, Pickrell J, Stephens M, Pritchard JK, Gilad Y: The contribution of RNA decay quantitative trait loci to inter-individual variation in steadystate gene expression levels. PLoS Genet 2012, 8(10):e1003000.

14. Jing Q, Huang S, Guth S, Zarubin T, Motoyama A, Chen J, Di Padova F, Lin S, Gram H, Han J: Involvement of MicroRNA in AU-rich element-mediated mRNA instability. Cell 2005, 120(5):623-634.

15. Sato F, Tsuchiya S, Meltzer S, Shimizu K: MicroRNAs and epigenetics. FEBS J 2011, 278(10):1598-1609.

16. Chuang J, Jones P: Epigenetics and microRNAs. Pediatr Res 2007, 61(5):24R-29R.

17. Costa F: Non-coding RNAs, epigenetics and complexity. Gene 2008, 410(1):9-17.

18. Drummond $D$, Raval A, Wilke $C$ : A single determinant dominates the rate of yeast protein evolution. Mol Biol Evol 2006, 23(2):327-337.

19. Valencia-Sanchez M, Liu J, Hannon G, Parker R: Control of translation and mRNA degradation by miRNAs and siRNAs. Genes Dev 2006, 20(5):515-524

20. Eisenberg $\mathrm{E}$, Levanon E: Human housekeeping genes are compact. Trends Genet 2003, 19(7):362-365.

21. Bergmiller T, Ackermann M, Silander O: Patterns of evolutionary conservation of essential genes correlate with their compensability. PLoS Genet 2012, 8(6):e1002803

22. Fabbri M, Calin G, Herceg Z, Ushijima T: Epigenetics and miRNAs in human cancer. Epigenetics Cancer Pt A 2010, 70:87-99.

23. Zhang Y, De S, Garner J, Smith K, Wang S, Becker K: Systematic analysis, comparison, and integration of disease based human genetic association data and mouse genetic phenotypic information. BMC Med Genomics 2010, 3:1.

24. Lewis B, Burge C, Bartel D: Conserved seed pairing, often flanked by adenosines, indicates that thousands of human genes are microRNA targets. Cell 2005, 120(1):15-20

25. Vergoulis T, Vlachos I, Alexiou P, Georgakilas G, Maragkakis M, Reczko M, Gerangelos S, Koziris N, Dalamagas T, Hatzigeorgiou A: TarBase 6.0: capturing the exponential growth of miRNA targets with experimental support. Nucleic Acids Res 2012, 40(D1):D222-D229.

26. Garcia D, Baek D, Shin C, Bell G, Grimson A, Bartel D: Weak seed-pairing stability and high target-site abundance decrease the proficiency of Isy-6 and other microRNAs. Nat Struct Mol Biol 2011, 18(10):1139-1146.

27. Dreszer T, Karolchik D, Zweig A, Hinrichs A, Raney B, Kuhn R, Meyer L, Wong M, Sloan C, Rosenbloom K, Roe G, Rhead B, Pohl A, Malladi VS, Li CH, Learned K, Kirkup V, Hsu F, Harte RA, Guruvadoo L, Goldman M, Giardine BM, Fujita PA, Diekhans M, Cline MS, Clawson H, Barber GP, Haussler D, James Kent W: The UCSC genome browser database: extensions and updates 2011. Nucleic Acids Res 2012, 40(D1):D918-D923.

28. Kuhn R, Haussler D, Kent W: The UCSC genome browser and associated tools. Brief Bioinform 2013, 14(2):144-161.

29. Flicek P, Ahmed I, Amode M, Barrell D, Beal K, Brent S, Carvalho-Silva D, Clapham P, Coates G, Fairley S, Fitzgerald S, Gil L, García-Girón C, Gordon L, Hourlier T, Hunt S, Juettemann T, Kähäri AK, Keenan S, Komorowska M, Kulesha E, Longden I, Maurel T, McLaren WM, Muffato M, Nag R, Overduin B, Pignatelli M, Pritchard B, Pritchard E, et al: Ensembl 2013. Nucleic Acids Res 2013, 41(D1):D48-D55.

30. Jacobsen A, Silber J, Harinath G, Huse J, Schultz N, Sander C: Analysis of microRNA-target interactions across diverse cancer types. Nat Struct Mol Biol 2013, 20(11):1325-1332.

31. Spasic M, Friedel C, Schott J, Kreth J, Leppek K, Hofmann S, Ozgur S, Stoecklin G: Genome-wide assessment of AU-rich elements by the AREScore algorithm. PLOS Genet 2012, 8(1):

32. Zhang Y, Lv J, Liu H, Zhu J, Su J, Wu Q, Qi Y, Wang F, Li X: HHMD: the human histone modification database. Nucleic Acids Res 2010, 38:D149-D154.

33. Hackenberg M, Barturen G, Oliver J: NGSmethDB: a database for nextgeneration sequencing single-cytosine-resolution DNA methylation data. Nucleic Acids Res 2011, 39:D75-D79.

34. Marcoulides $\mathrm{G}$ : The elements of statistical learning: data mining, inference and prediction. Struct Equ Modeling 2004, 11(1):150-151.

doi:10.1186/1471-2164-15-1010

Cite this article as: Das et al:: Insights into the miRNA regulations in human disease genes. BMC Genomics 2014 15:1010. 